Pacific Journal of Mathematics

A COMMUTATIVITY STUDY FOR PERIODIC RINGS 


\title{
A COMMUTATIVITY STUDY FOR PERIODIC RINGS
}

\author{
Howard E. Bell
}

Putcha and Yaqub have proved that a ring $R$ satisfying a polynomial identity of the form $x y=\omega(x, y)$, where $\omega(X, Y)$ is a word different from $X Y$, must have nil commutator ideal. Our first major theorem extends this result to the case where $\omega(X, Y)$ varies with $x$ and $y$, with the restriction that all $\omega(X, Y)$ have length at least three and are not of the form $X^{n} Y$ or $X Y^{n}$. Further restrictions on the $\omega(X, Y)$ are then shown to yield commutativity of $R$; among these is a semigroup condition of Tamura, Putcha, and Weissglass - sepecifically, that each $\omega(X, Y)$ begins with $Y$ and has degree at least 2 in $X$. The final theorem establishes commutativity of rings $R$ satisfying $x y=$ $y x s$, where $s=s(x, y)$ is an element in the center of the subring generated by $x$ and $y$. All rings considered are either periodic by hypothesis or turn out to be periodic in the course of the investigation.

\section{Definitions and preliminary results. Let $\omega=$} $\omega(X, Y)$ be a word or monomial in the noncommuting indeterminates $X$ and $Y$; that is, $\omega$ is a polynomial of form

$$
Y^{\jmath_{1}} X^{k_{1}} Y^{\jmath_{2}} X^{k_{2}} \cdots Y^{\jmath_{s}} X^{k_{s}}
$$

where $j_{l}, k_{\imath} \geqq 0$ for $i=1, \cdots, s$ and $\sum_{i=1}^{s}\left(j_{1}+k_{t}\right)>0$. By the $X$-length (resp. $Y$-length) of $\omega$, which we denote by $|\omega|_{X}$ (resp. $|\omega|_{Y}$ ), we shall mean the non-negative integer $\Sigma k_{\imath}$ (resp. $\Sigma j_{l}$ ); the sum $|\omega|_{X}+|\omega|_{Y}$ will be called the length of $\omega$ and denoted by $|\omega|$. It will be convenient to divide the set of all words into nine types as follows:

(i) words with $|\omega|_{X} \geqq 2$ and $|\omega|_{Y} \geqq 2$;

(ii) words of form $Y X^{n}, n \geqq 1$;

(iii) words of form $Y^{n} X, n \geqq 1$;

(iv) words with $|\omega|_{Y}=0$;

(v) words with $|\omega|_{X}=0$;

(vi) words of form $X^{n} Y X^{m}, n, m \geqq 1$;

(vii) words of form $Y^{n} X Y^{m}, n, m \geqq 1$;

(viii) words of form $X^{n} Y, n \geqq 1$;

(ix) words of form $X Y^{n}, n \geqq 1$.

A word of form (1) having $j_{1} \geqq 1$ and $|\omega|_{X} \geqq 2$ will be called a Tamura-Putcha-Weissglass $(T-P-W)$ word; a word which is either $Y X$ or a $T-P-W$ word will be called a $G-T-P-W$ word. A multiplicative semigroup $S$ will be called a $T-P-W$ (resp. $G-T-P-W$ ) semigroup if for 
each $x, y \in S$, there exists a $T-P-W$ (resp. $G-T-P-W$ ) word $\omega$ for which $x y=\omega(x, y)$.

A ring $R$ will be called periodic if for each $x \in R$, there exist distinct positive integers $n, m$, depending on $x$, for which $x^{n}=x^{m}$. Among the periodic (in fact, finite) rings which we shall refer to frequently are the Corbas $(p, k, \phi)$-rings [5], which we define as follows: $R^{+}$is the additive direct-sum $G F\left(p^{k}\right) \oplus G F\left(p^{k}\right), \phi$ is an automorphism of $G F\left(p^{k}\right)$, and ring multiplication is defined by

$$
(a, b)(c, d)=(a c, a d+b \phi(c)) .
$$

These rings have the property that $D^{2}=0$, where $D$ denotes the set of zero divisors (including 0); and they have as few zero divisors as a non-domain may have-specifically, $|D|^{2}=|R|$ [5]. They are commutative rings only when $\phi$ is the identity automorphism.

We shall make repeated use of two basic theorems on periodic rings. The second is a special case of an old theorem of Herstein; but since he deduces it as a corollary of one of his more complicated commutativity theorems, we think it worthwhile to include a simple proof.

LEMMA 1. If $R$ is any periodic ring, then $R$ has each of the following properties:

(a) For each $x \in R$, some power of $x$ is idempotent.

(b) For each $x \in R$, there exists an integer $n(x)>1$ such that $x-x^{n(x)}$ is nilpotent.

(c) Each $x \in R$ can be expressed in the form $y+w$, where $y^{n}=y$ for some $n=n(y)>1$ and $w$ is nilpotent.

(d) If $I$ is an ideal of $R$ and $x+I$ is a nonzero nilpotent element of $R / I$, then $R$ contains a nilpotent element $u$ such that $x \equiv u(\bmod I)$.

Proof. (a) If $x^{n}=x^{m}$ with $n>m$, then $x^{j+k(n-m)}=x^{j}$ for each positive integer $k$ and each $j \geqq m$; thus, we may assume $n-m+1 \geqq$ $m$. It follows that $x^{n-m+1}=\left(x^{n-m+1}\right)^{n-m+1}$ and hence $\left(x^{n-m+1}\right)^{n-m}$ is idempotent.

(b) Let $x^{n}=x^{m}, n>m>1$. Then

$$
x^{m-1}\left(x-x^{n-m+1}\right)=0=x^{m-2} x\left(x-x^{n-m+1}\right)=x^{m-2} x^{n-m+1}\left(x-x^{n-m+1}\right) ;
$$

therefore, $x^{m-2}\left(x-x^{n-m+1}\right)^{2}=0$ and the result follows by the obvious induction.

(c) If $x^{n}=x^{m}$ with $n \geqq n-m+1>m$, the proofs of (a) and (b) show that we may take $y=x^{n-m+1}$ and $w=x-x^{n-m+1}$.

(d) If $x+I$ is a nonzero nilpotent element of $R / I$, there exists a 
positive integer $k$ such that $x^{q} \in I$ for all $q \geqq k$. By the proofs of (a) and (b), $R$ contains a nilpotent element $u=x-x^{q}$ with $q \geqq k$; clearly, $u \equiv x$ (mod. I).

THEOREM 2. (Herstein, [8]) If $R$ is a periodic ring with all nilpotent elements central, then $R$ is commutative.

Proof. Let $N$ denote the set of nilpotent elements; the usual argument for commutative rings shows that $N$ is an ideal. Moreover, for $x \in R$ and $e$ an idempotent in $R$, both ex - exe and $x e$-exe are in $N$, hence commute with $e$; thus, idempotents in $R$ are central.

By (d) of Lemma 1, we see that homomorphic images inherit the hypotheses on $R$; consequently, we need consider only the case of subdirectly irreducible $R$. Under this assumption, part (a) of Lemma 1 shows that $R$ is either nil and hence commutative, or $R$ has a unique nonzero central idempotent, necessarily a multiplicative identity element 1 .

Considering this latter possibility, we see from (a) of Lemma 1 that each element of $R$ is either nilpotent or invertible; thus, the set $D$ of zero divisors is equal to $N$ and hence is a central ideal. Moreover, by Lemma (b), $\bar{R}=R / D$ has the $a^{n}=a$ property of Jacobson; hence $\bar{R}$ is commutative and its additive group is a torsion group. Thus, if $a$, $b \in R \backslash D$, the subring of $\bar{R}$ generated by $\bar{a}=a+D$ and $\bar{b}=b+D$ is a finite field, which has cyclic multiplicative group. There must therefore exist $g \in R$ and $d_{1}, d_{2} \in D$ such that $a=g^{1}+d_{1}$ and $b=g^{j}+d_{2}$ for some positive integers $i, j$. It follows that $a$ and $b$ must commute, and our proof is complete.

\section{A nil-commutator-ideal theorem and some} relatives.

THEOREM 3. Let $R$ be a ring such that for each $x, y \in R$, there exists a word $\omega(X, Y)$, of one of the types (i)-(vii) and with $|\omega| \geqq 3$, for which $x y=\omega(x, y)$. Then the set $N$ of nilpotent elements forms an ideal, and the commutator ideal $C(R)$ is contained in $N$.

Proof. Taking $x=y$ shows that for each $x \in R, x^{2}=x^{k}$ for some $k>2$; hence $R$ is periodic and each nilpotent element squares to zero. We next show that idempotents of $R$ must be central. Let $e$ be a non-zero idempotent, let $x \in R$, and suppose $\omega(X, Y)$ is a word of the allowed types for which $e(e x-e x e)=\omega(e, e x-e x e)$. Clearly, $\omega$ cannot be of type (iv) since $(e x-e x e)^{2}=0$; and any of the other types has either two adjacent $Y$ 's or a $Y$ preceding an $X$. Thus $e(e x-e x e)=$ $e x-e x e=0$, and similarly $x e-e x e=0$. 
It is proved in [3] that a periodic ring satisfies the conclusions of the theorem if nilpotent elements commute with each other, so we may complete our proof by showing that $x y=0$ for all $x, y \in N$. Accordingly, let $x, y \in N$ and $\omega$ a word such that $x y=$ $\omega(x, y)$. If $\omega$ has two adjacent $X$ 's or $Y$ 's, then it is immediate that $x y=0$; otherwise, we have one of the following cases: (a) $x y=(x y)^{k}$ for some $k>1$; (b) $x y=x y x y \cdots x$; (c) $x y=y x y \cdots$. In case (a), $(x y)^{k-1}$ is idempotent, hence central; and we get $x y=x(x y)^{k-1} y=0$. In case (b) right-multiplication by $x$ yields $x y x=0=x y$, and in case (c) leftmultiplication by $y$ yields $y x y=0=x y$.

Remarks. An alternative, somewhat deeper, method of proof is to note that idempotents are central, apply (a) of Lemma 1 to show that some power of each element is central, and appeal to a well-known theorem of Herstein [7].

In the hypotheses of Theorem 3, the restriction on the type of $\omega(X, Y)$ is essential, for without it, as Putcha and Yaqub have pointed out in [11], the ring of $2 \times 2$ matrices over $G F(2)$ would satisfy the hypotheses.

The hypotheses of Theorem 3 will not yield commutativity of $R$. The Corbas $(2,2, \phi)$-ring is a counterexample, where $\phi$ is the nonidentity automorphism of $\mathrm{GF}(4)$-indeed, in this ring, if $u, v \in N$ and $x, y \notin N$, we have $u v=v u^{2}, x u=u x^{2}, u x=x u x^{2}$, and $x y=$ $(y x)^{3} x y$. However, restriction of $\omega(X, Y)$ to words of fixed type (i)-(vii) does yield commutativity, as we now prove.

THEOREM 4. Let $\alpha$ denote a fixed one of the word-types (i)-(vii). Let $R$ be a ring such that for each $x, y \in R$, there exists a type- $\alpha$ word $\omega(X, Y)$, depending on $x$ and $y$ and having length at least three, for which $x y=\omega(x, y)$. Then $R$ is commutative.

Proof. If $\alpha$ is type (i), commutativity follows from a theorem of Putcha and Yaqub [12]; types (ii) and (iii) are covered by a theorem of the present author [1, 2]. Suppose, then, that $\alpha$ is type (iv), i.e. for each $x$, $y \in R, x y=x^{n}$ for some $n=n(x, y) \geqq 3$. Then, since nilpotent elements square to 0 , they left-annihilate $R$. Taking $x \in N$ and $a$ an element such that $a^{k}=a, k>1$, and recalling that idempotents are central, we obtain the result that $a x=a a^{k-1} x=a x a^{k-1}=0$; and by (c) of Lemma 1, nilpotent elements right-annihilate $R$ as well and commutativity follows from Theorem 2. Clearly, type (v) may be treated similarly.

To complete the proof, we discuss type (vi), noting that (vii) is similar. Let $x \in N, y \in R$ and $x y=x^{n} y x^{m}$, with $n, m \geqq 1$. If either of $n, m$ is greater than 1 , then $x y=0$; if $x y=x y x$, right-multiplying by $x$ yields $x y x=0=x y$. Also, $y x=y^{j} x y^{k}$ with $k \geqq 1$, so $y x=0$ as well, and again commutativity follows by Theorem 2 . 
THEOREM 5. Suppose that for each $x, y \in R$, there exists an integer $n(x, y)>1$ such that $x y=x^{n(x, y)} y$. Then the commutator ideal $C(R)$ is nil and the nilpotent elements form an ideal. If the idempotents of $R$ are central, then $R$ is commutative.

Proof. Clearly $R$ is periodic with nilpotent elements squaring to zero, and for $x \in R$ and $u$ nilpotent we have $u x=u^{n} x=0$. Thus the set $N$ of nilpotent elements is the left annihilator of $R$, hence an ideal. The ring $R / N$ has the $a^{n}=a$ property by Lemma 1 (b), hence is commutative. Thus $C(R) \subseteq N$.

Now assume that idempotents are central. If $a^{k}=a$ for $k>1$, and $u \in N$, we get $a u=a^{n} u=a^{n-1} a a^{k-1} u=a^{n} u a^{k-1}=0$; hence by Lemma 1 (c) and Theorem $2, R$ is commutative.

Remarks. Centrality of idempotents is not implied by the condition $x y=x^{n} y$. A counterexample is the ring $R$ with additive group equal to the Klein 4-group and multiplication given by $0 x=c x=0$ and $a x=b x=x$ for all $x \in R$; this ring satisfies the identity $x y=x^{2} y$.

In the event that idempotents are central in Theorem 5 , we can say a bit more about $R$ - specifically, it is the direct sum of a zero ring and a $J$-ring (i.e. one with Jacobson's $a^{n}=a$ property). For if $x, y$ are arbitrary elements of $R$, there exist integers $n_{1}, n_{2}>1$ such that $x y=x^{n_{1}} y$ and $y x=y^{n_{2}} x$. A standard computation yields a single $n$ such that $x y=x^{n} y$ and $y x=y^{n} x$, and the commutativity now shows that $x^{n} y=$ $x y^{n}$. The direct-sum decomposition of rings with the latter type of constraint has essentially been obtained in [9] and [15]. (Actually those papers assume $n$ constant, but the extension to variable $n$ is not difficult.)

\section{Two commutativity theorems.}

THEOREM 6. Let $R$ be a periodic ring, the multiplicative semigroup of which is a G-T-P-W semigroup. Then $R$ is commutative.

Proof. If $a, b \in R$ and $a b=0$, then $b a=0$ also. This observation implies that the nilpotent elements of $R$ form an ideal $N$, which, since $R$ is periodic, must coincide with the Jacobson radical $J(R)$.

Again we wish to deduce our result from Theorem 2. Suppose, then, that $v$ is a noncentral nilpotent element and $b \in R$ is an element not commuting with $v$. Then

$$
v b=b^{\prime \prime} v^{k_{1}} \cdots v^{k_{s}} \quad \text { with } \quad j_{1} \geqq 1 \text { and } \Sigma k_{i} \geqq 2 .
$$

If $k_{1} \geqq 2$, we obtain

$$
v b=b^{h_{1}} v v^{k_{1}-1} \cdots v^{k_{s}}=v^{t}\left(b^{j_{1}}\right)^{q} \cdots v^{k_{1}-1} \cdots v^{k_{s}} .
$$


If $t=1$, we make no further substitutions in (4); otherwise, we write $v b=v v^{t-1} b^{j_{1} q} \cdots v^{k_{1}-1} \cdots v^{k_{s}}=v b^{i_{1} q z}\left(v^{t-1}\right)^{n} \cdots v^{k_{s}}$. In either case, we have $v b=v b y$ for some $y \in J(R)$, from which it follows that $v b=0=b v$, contradicting our choice of $v$. If $k_{1}=1$ in (3), then some other $k_{i}$ is positive, and a similar computation again yields the same contradiction. Thus, nilpotent elements of $R$ are central, and our proof is complete.

COROLlaRY 7. Let $R$ be any ring having as multiplicative semi. group a T-P-W semigroup. Then $R$ is commutative.

Note that Theorem 6 and Corollary 7 would not be true if the condition $|\omega|_{X} \geqq 2$ were omitted from the definition of $G-T-P-W$ and $T-P-W$ words - again the Corbas $(2,2, \phi)$-ring is the revealing example.

THEOREM 8. Let $R$ be any ring such that for each $x, y \in R$, there exists an element $s=s(x, y)$ in the center of the subring generated by $x$ and $y$, for which $x y=y x$ s. Then $R$ is commutative.

Proof. Taking $x=y$ shows that $x^{2}=x^{2} p(x)$, where $p(x)$ is a polynomial with integer coefficients and zero constant term; it follows by a theorem of Chacron [4] that $R$ is periodic. Moreover, the given constraint shows that $a b=0$ implies $b a=0=a r b$ for arbitrary $r \in R$. This result, together with the obvious fact that nilpotent elements square to zero, shows that $u v s=0$ for any nilpotent $u$ and $v$ and any $s$ in the subring generated by $u$ and $v$; thus, the nilpotent elements form an ideal $N$ with $N^{2}=0$. Moreover, a standard argument applied to $e$, ex - exe, and $x e$-exe shows that all idempotents $e$ are central.

The hypotheses of the theorem persist under the taking of homomorphic images, so we need consider only subdirectly irreducible $R$. Since nil rings with our condition are zero rings, and since subdirectly irreducible rings can have at most one nonzero central idempotent, Lemma 1(a) allows us to assume that $R$ has 1 and that every nonnilpotent element is invertible. Hence the set $D$ of zero divisors is an ideal, equal to $N$.

Since there exist distinct $n, m$ with $(1+1)^{n}=(1+1)^{m}, R^{+}$must be a torsion group, which in view of subdirect irreducibility, is a $p$-group for some prime $p$. Since $D^{2}=0$, we then have $(p \cdot 1)(p x)=p^{2} x=0$ for all $x \in R$.

Now $R$ is clearly a duo ring, so we may apply Thierrin's results on subdirectly irreducible duo rings [14]. Specifically, letting $S$ denote the intersection of the nonzero ideals of $R$ and noting that $R \neq D$, we have $S$ equal to the annihilator of $D$-that is, $S=D$. By Lemma 1 (b) and the " $a{ }^{n}=a$ theorem" we know that $R / D$ is commutative, and hence that 
commutators in $R$ belong to $D$. Suppose now that $p R \neq 0$, let $p x \neq 0$, and let $y$ be an arbitrary element of $R$. Since $p x R$ is a nonzero ideal, we have $x y-y x \in D=S \subseteq p x R$, and there exists $r \in R$ such that $x y-y x=p x r$ and hence $p(x y-y x)=p^{2} x r=0$. Thus $p R=D$ is central, and commutativity of $R$ follows from Theorem 2 .

Now suppose that we have a subdirectly irreducible counterexample with $p R=0$. Applying Lemma $1(\mathrm{c})$ and the fact that $D^{2}=0$, we can then choose a non-central nilpotent element $u$ and an element $b \in R$ such that $b^{n(b)}=b$ for some $n(b)>1$ and $b$ does not commute with $u$. Since $b u=u b s$ for some $s$ in the subring generated by $u$ and $b$, and since $u r u=0$ for all $r \in R$, we obtain $b u=u b p(b)$, where $p(X)$ is some polynomial with integer coefficients and zero constant term. It follows that the subring $\langle u, b\rangle$ of $R$ generated by $u$ and $b$ is finite. Since the hypotheses of the theorem are inherited by subrings and by homomorphic images, we can conclude that some homomorphic image $T$ of $\langle u, b\rangle$ is a finite subdirectly irreducible counterexample with $p T=0$.

As in [2], we can argue that $T$ must be a Corbas $(p, k, \phi)$-ring for appropriate choices of $p, k$, and $\phi$. Indeed, Corbas showed in [6] that finite rings $R$ with 1 and with $D^{2}=0=p R$ must have additive group which is a direct sum $K \oplus D$, where $K$ is a finite field and $D$ is a left vector space over $K$. Since one-dimensional subspaces of $D$ are left ideals, the fact that our $T$ is subdirectly irreducible and a duo ring shows that $D$ is one-dimensional and $|T|=|D|^{2}$; and we apply an earlier result of Corbas [5] to show that $T$ is a $(p, k, \phi)$-ring.

Consider any Corbas $(p, k, \phi)$-ring $T$ with $\phi$ a nonidentity automorphism of $K=G F\left(p^{k}\right)$; let $g$ be a generator of the multiplicative group of $K$, and let $\phi$ be given by $x \rightarrow x^{p^{r}}, 1 \leqq r<k$. If $(a, b) \in T$ commutes with both $(g, 0)$ and $(0, g)$, then by (2) we have $b=0$ and $a=\phi(a)$. Then imposing the condition that $(g, 0)(0, g)=(0, g)(g, 0)(a, 0)$ yields $g=\phi(g) a$. Since $\phi(g)=g^{p^{r}}$ and $g=g^{p^{k}}$, we have $g^{p^{k}}=$

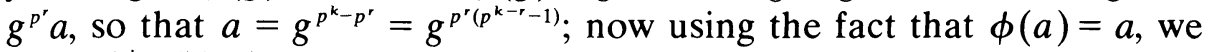

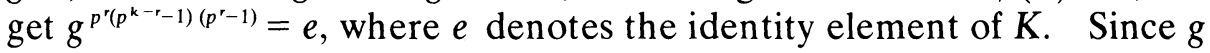
has order $p^{k}-1$, which is relatively prime to $p^{r}$, we conclude that $p^{k}-1 \mid\left(p^{k-r}-1\right)\left(p^{r}-1\right)$, which is absurd. The possibility of a counterexample is thus demolished, and the proof is complete.

REMARK. It is tempting to conjecture that $R$ must be commutative if it satisfies $x y=y x s$, where $s=s(x, y)$ is merely assumed to belong to the subring generated by $x$ and $y$ and not necessarily to its center. However, the Corbas $(2,2, \phi)$-ring shows that this is not true.

\section{REFERENCES}

1. H. E. Bell, Some commutativity results for rings with two-variable constraints, Proc. Amer. Math. Soc., 53 (1975), 280-284. 
2. - A commutativity condition for rings, Canad. J. Math., 28 (1976), 986-991.

3. _-_ Some commutativity results for periodic rings, Acta Math. Acad. Sci. Hungar., 28 (1976), 279-283.

4. M. Chacron, On a theorem of Herstein, Canad. J. Math., 21 (1969), 1348-1353.

5. B. Corbas, Ring with few zero divisors, Math. Ann., 181 (1969), 1-7.

6. - Finite rings in which the product of any two zero divisors is zero, Arch. Math., 21 (1970), 466-469.

7. I. N. Herstein, A theorem on rings, Canad. J. Math., 5 (1953), 238-241.

8. - A note on rings with central nilpotent elements, Proc. Amer. Math. Soc., 5 (1954), 620.

9. J. Luh, On the structure of pre- $J$-rings, Hung-ching Chow Sixty-fifth Anniversary Volume, 47-52. Math. Res. Center Nat. Taiwan Univ., Taipei 1967. MR37 \#250.

10. M. S. Putcha and J. Weissglass, Semigroups satisfying variable identities, Semigroup Forum, 3 (1971), 64-67.

11. M. S. Putcha and A. Yaqub, Rings satisfying monomial identities, Proc. Amer. Math. Soc., 32 (1972), 52-56.

12. - Structure of rings satisfying certain polynomial identities, J. Math. Soc. Japan, 24 (1972), 123-127.

13. T. Tamura, Semigroups satisfying identity $x y=f(x, y)$, Pacific J. Math., 31 (1969), 513-521.

14. G. Thierrin, On duo rings, Canad. Math. Bull., 3 (1960), 167-172.

15. A. Yaqub, The structure of pre- $p^{k}$-rings and generalized pre-p-rings, Amer. Math. Monthly, 71 (1964), 1010-1014.

Received December 9, 1976 and in revised form March 3, 1977. Supported by the National Research Council of Canada, Grant No. A3961.

Brock University St. Catherines, Ontario, Canada 



\section{Pacific Journal of Mathematics \\ Vol. 70, No. $1 \quad$ September, 1977}

William H. Barker, Noether's theorem for plane domains with hyperelliptic

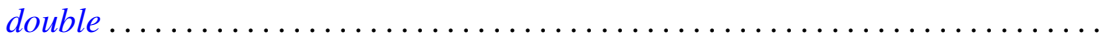

Michael James Beeson, Non-continuous dependence of surfaces of least area on the boundary curve ...................................... 11

Horst Behncke, Functions acting in weighted Orlicz algebras . . . . . . . . . . . . 19

Howard Edwin Bell, A commutativity study for periodic rings . . . . . . . . . . . 29

Peter Botta and Stephen J. Pierce, The preservers of any orthogonal group ....... 37

Douglas S. Bridges, The constructive Radon-Nikodým theorem ............. 51

James Dennis Brom, The theory of almost periodic functions in constructive

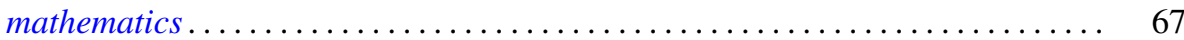

N. Burgoyne and C. Williamson, Semi-simple classes in Chevalley type groups ....

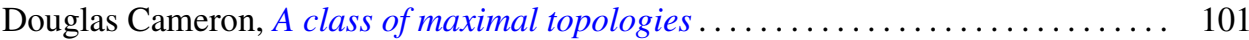

L. Carlitz, Enumeration of doubly up-down permutations . . . . . . . . . . . . . . 105

Paul Robert Chernoff, The quantum n-body problem and a theorem of

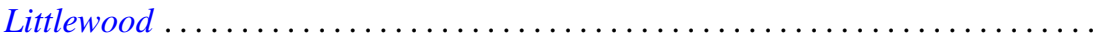

Jo-Ann Deborah Cohen, Locally bounded topologies on $F(X) \ldots \ldots \ldots \ldots \ldots \ldots$

Heinz Otto Cordes and Robert Colman McOwen, Remarks on singular elliptic

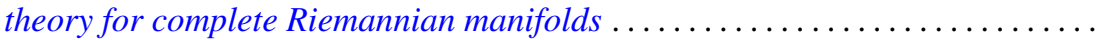

Micheal Neal Dyer, Correction to: "Rational homology and Whitehead

products"

Robert Fernholz, Factorization of Radonifying transformations

Lawrence Arthur Fialkow, A note on quasisimilarity. II ...... . .

Harvey Charles Greenwald, Lipschitz spaces of distributions on the surface of unit

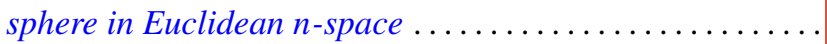

Albrecht Irle, On the measurability of conditional expectations

Tom (Roy Thomas Jr.) Jacob, Matrix transformations involving simple sequence

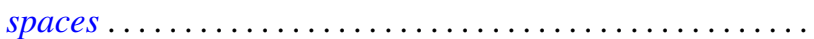

A. Katsaras, Continuous linear maps positive on increasing continuous

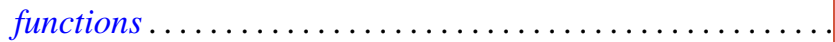

Kenneth Kunen and Judith Roitman, Attaining the spread at cardinals of cofinality

Lawrence Louis Larmore and Robert David Rigdon, Enumerating normal bundles

of immersions and embeddings of projective spaces ...... . .

Ch. G. Philos and V. A. Staïkos, Asymptotic properties of nonoscillatory solutions of differential equations with deviating argument .

Peter Michael Rosenthal and Ahmed Ramzy Sourour, On operator algebras containing cyclic Boolean algebras...

Polychronis Strantzalos, Strikt fast gleichgradig-stetige und eigentliche

Aktionen ...

Glenn Francis Webb, Exponential representation of solutions to an abstract

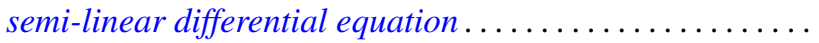

